\title{
Serological evidence of chikungunya and malaria co-infection among febrile patients seeking health care in Karagwe district, Tanzania
}

\author{
EDSON KINIMI ${ }^{*}$, BISIMWA N. PATRICK 3 and GERALD MISINZO² \\ 'Department of Veterinary Physiology, Biochemistry and Pharmacology, College of Veterinary Medicine and \\ Biomedical Sciences, Sokoine University of Agriculture, P. O. Box 3017, Morogoro, Tanzania. \\ ${ }^{2}$ Southern African Centre for Infectious Disease Surveillance - Africa Centre of Excellence for Infectious Disease \\ of Human and Animals, Sokoine University of Agriculture, P.O. Box 3297, Morogoro, Tanzania \\ ${ }^{3}$ Department of Animal Science, Faculty of Agricultural and Environmental Studies, Université Evangélique en \\ Afrique, P.O. Box 3323, Bukavu, Democratic Republic of Congo
}

\begin{abstract}
Background: Chikungunya is an emerging mosquito-borne viral illness of major public health concern and is becoming a common infection in many geographical areas of Tanzania. This study was carried out to determine the incidence of malaria and chikungunya infections among febrile patients seeking medical care in Karagwe district, Tanzania.

Methods: Febrile patients were enrolled into the study at Nyakahanga district designated hospital and Kayanga heath centre in May and June 2015. Questionnaires were administered to collect clinical and socio-demographic characteristics of patients. All participants were tested for malaria using malarial rapid diagnostic test and those tested positive by mRDT were confirmed by microscopy. Both outpatients tested malaria positive and negative were further screened for immunoglobulin $M(\operatorname{Ig} M)$ and G (IgG) antibodies for chikungunya using enzyme-linked immunosorbent assay.

Results: A total of 400 febrile patients were enrolled in the study. Out of 400 febrile outpatients tested for malaria, 116 (28.75\%) tested positive with mRDT. Microscopy confirmed presence malaria parasites in 112 (96.55\%) of the malaria RDT-positive. The overall seroprevalence of chikungunya infection was $24.25 \%$ (97/400). Out of those chikungunya seropositive subjects, 89 (91.75\%) had no malaria. Co-infection rate of chikungunya and malaria was found to be $7.14 \%$ (8/112).

Conclusions: Our findings confirmed the existence of chikungunya and malaria co-infection among febrile patients seeking health care in Karagwe district. Chikungunya should be considered in the differential diagnosis of malaria for appropriate case management and in order to monitor the public health burden and to inform possible preventative and control measures.
\end{abstract}

Keywords: Chikungunya, malaria, co-infection, seroprevalence, Tanzania

\section{Introduction}

Chikungunya is a mosquito-borne virus infection transmitted to humans through the bite of infected mosquitoes of the genus Aedes. Aedes aegypti and Aedes albopictus are the primary vectors of Chikungunya virus (CHIKV), but also the virus is maintained in sylvatic cycles involving primates and forest dwelling Aedes species (Deller \& Russell, 1968; Mohan et al., 2010; Thiberville et al., 2013). The CHIKV (genus Alphavirus, family Togaviridae) was first recognized and isolated in 1952 from Makonde Plateau in southern Tanzania (Robinson, 1955). The word "chikungunya" originates from Makonde language referring to the bending stance in afflicted patients due to severe musculoskeletal pain (Lumsden, 1955; Hertz et al., 2012; Thiberville et al., 2013).

Chikungunya is currently of a global public health concern due to its continued spread and escalating epidemic trends throughout the tropical and subtropical regions of the world (Deller \& Russell, 1968; Mohan et al., 2010). This is attributed to the expansion of the vectors' geographical range, global travel, unplanned urbanization and climate change (Thiberville et al., 2013). Since the 1960s, CHIKV has repeatedly been isolated in Africa, Asia and Latin America. In Africa, chikungunya outbreaks have been reported in 25 countries (CDC, 2016). In Tanzania, chikungunya has been reported in southern, south-western, northern and central regions of the country (Robinson, 1955;

\footnotetext{
* Correspondence E-mail: kinimiesdon@gmail.com
} 
Hertz et al., 2012; Crump et al., 2013; Weller et al., 2014; Chipwaza et al., 2014; Kajeguka et al., 2016; Faustine et al., 2017).

Malaria is the primary cause of fever in all age groups and contributes to considerable mortalities in Sub Saharan Africa including Tanzania (Mboera et al., 2007). In Tanzania, malaria is primarily transmitted by female Anopheles gambiae, An. arabiensis and An. funestus. Four malaria causative agents (plasmodia species), namely Plasmodium falciparum, $P$. vivax, $P$. malariae, and $P$. ovale are prevalent in the country (Mboera et al., 2013). Chikungunya may occur concurrently with malaria and non-malaria febrile illnesses (Sow et al., 2016). Co-infection of chikungunya and malaria is possible in geographical locations where the vectors co-exist (Sow et al., 2016). As the species of mosquitoes responsible for transmission of these infections are present in Tanzania (Ndosi et al., 2016; Bisimwa et al., 2018), co-infection is expected to occur.

Tanzania is experiencing a change in aetiologies of febrile illnesses as it is for many other African countries. While the numbers of malarial febrile illnesses are decreasing in sub-Saharan Africa (O'Meara et al., 2008; Mboera et al., 2013), the numbers of non-malarial febrile cases are increasing (Mtove et al., 2006; Ligon, 2006; Hercik et al., 2017). Arboviruses are considered the likely cause of some non-malarial febrile illnesses and are gaining more prominence as observed through increased mosquito-borne viral disease epidemics (Crump et al., 2013). Patients with nonmalarial febrile illnesses such as chikungunya may present with clinical signs similar to those of other febrile illnesses, thus laboratory confirmation is essential but often lacking (D'Acremont et al.,2014). Recent studies in Tanzania have revealed that patients with acute dengue and chikungunya infections are often misdiagnosed and treated with antimalarials or antibiotics, mainly due to lack of differential diagnosis (Chipwaza et al., 2014). Screening of aetiologies of fever cases which occur concurrently with malaria is not commonly practiced in most health facilities in Tanzania. This study aimed to determine the seroprevalence of chikungunya and malaria coinfections among febrile patients seeking medical care in Karagwe district of north-western Tanzania.

\section{Materials and Methods}

\section{Study Site}

This study was carried out in Karagwe district, located in Kagera region in north-western Tanzania. The district is characterized by mountain ranges with swampy valley bottoms and wetlands. The altitude ranges between 1,500 and 1,800 meters above sea level. The annual average temperature is $26^{\circ} \mathrm{C}$. Rainfall distribution is bi-modal with peaks between September-December and MarchMay. Most parts of Karagwe district receive average rainfall between $800 \mathrm{~mm}$ and $1000 \mathrm{~mm}$ annually. The major economic activity of the majority of the population in the district is agriculture with banana, coffee and maize being the main crops. Similarly, inhabitants are involved in rural subsistence fishing and livestock keeping. Karagwe district has experienced malaria epidemics for many years with unstable transmission of varying seasonality (Mboera \& Kitua, 2001; Mashauri et al., 2013). Significant malaria epidemic was recorded in 1997/1998, which was associated with unusually heavy El Nino-Southern Oscillation (ENSO) rains (Carlstedt, 1997; Lindsay et al., 2000).

\section{Study design}

This facility based study involved Nyakahanga District Designated Hospital and Kayanga Heath Centre and was carried out in May and June 2015. It involved outpatients presenting with high fever (axillary or rectal temperature $\geq 38^{\circ} \mathrm{C}$ ) at the facilities. Participants who had recent or in evidence of previous fever 2-5 days and other clinical manifestations such as headache, skin rashes, joint ache, muscle and body pains were also considered. Thus, febrile patients who gave their consent were enrolled into the study. Exclusion criteria included participants with chronic diseases, which required instant medical intervention. 


\section{Data collection}

Whole blood samples were obtained from the febrile patients by venipuncture and transferred into plain vacutainer tubes. Few drops of whole blood (2-3drops) were used for malaria parasite detection (mRDT), approximately $18 \mu$ l of which was used to prepare blood smear (BS) for malaria parasite identification. The remainder of the whole blood sample from each patient was centrifuged and the sera were used for chikungunya serological tests. The blood smears were stained with $20 \%$ Giemsa stain for plasmodium parasite identification. A structured questionnaire was administered to collect socio-demographic and clinical data. Upon recruitment of eligible patients, the clinician documented sex, age, clinical manifestations of the patient and date of onset of disease.

\section{Serological assays}

Malarial rapid diagnostic test (mRDT-Paracheck $\mathrm{Pf}^{\oplus}$, Orchid Biomedical system) was used to test presence of malaria antigens at the health facility. Serum sample from each patient was tested for anti- CHIKV IgM and IgG antibodies using ELISA (EUROIMMUN AG, Lüebeck, Germany) at the College of Veterinary Medicine and Biomedical Sciences of the Sokoine University of Agriculture. The results of mRDT and ELISA serologic analyses were interpreted according to the manufacturers' protocol.

\section{Data analysis}

The statistical analysis was performed using Microsoft Office-Excel 2007 (Microsoft, USA) and Epi Info version 7.0.8.0 (CDC, Atlanta, USA). Data on proportions were compared using Chi Square. All tests were performed at $\mathrm{P} \leq 0.05$ and were considered statistically significant.

\section{Ethical considerations}

The study was approved by the Medical Research Coordinating Committee of the National Institute for Medical Research, Tanzania (Approval number NIMR/HQ/R.8a/Vol.1X/1946). Questionnaire and laboratory results were stored separately under unique study identity number without any biodata. All minors were included in the study only if their parent/guardian provided consent.

\section{Results}

\section{The demographic and clinical profiles of enrolled outpatients}

A total of 400 febrile patients composed of 152 (38.00\%) males and $248(62.00 \%)$ females were enrolled. Most of febrile subjects were between 20 and 29 years (25.25\%), followed by those aged $10-19$ years $(23.25 \%)$, and only $14.00 \%$ were at least 50 years old (Table 1 ). The most common symptoms and signs exhibited among febrile patients were headache (69.00\%), joint aches (68.25\%), Vomiting (61.50\%), leg weaknesses (59.50\%) and laboured breathing (58.00\%).The least observed symptoms were abdominal pains (41.75\%), neck stiffness (33.75\%), and skin rashes (33.00\%), (Table 2).

Table 1: Age and sex distribution of the enrolled participants

\begin{tabular}{|c|c|c|c|c|c|c|}
\hline $\begin{array}{l}\text { Age } \\
\text { (Year) }\end{array}$ & $\begin{array}{l}\text { Enrolled subjects } \\
(\%) n=400\end{array}$ & $\begin{array}{l}\text { Male } \\
\mathrm{n}=152\end{array}$ & $\begin{array}{l}\text { Female (\%) } \\
\mathrm{n}=248\end{array}$ & $\begin{array}{l}\text { CHIK } \\
n=97\end{array}$ & $\begin{array}{l}\text { Mal } \\
\mathrm{n}=112\end{array}$ & $\begin{array}{l}\mathrm{CHIK}+\mathrm{MAL} \\
\mathrm{n}=8\end{array}$ \\
\hline $1-9$ & $48(12.00)$ & $15(9.87)$ & $33(13.31)$ & 6 & 9 & 0 \\
\hline $10-19$ & $93(23.25)$ & $39(25.66)$ & $54(21.77)$ & 8 & 14 & 0 \\
\hline $20-29$ & $101(25.25)$ & $52(34.21)$ & $49(19.76)$ & 52 & 37 & 6 \\
\hline $30-39$ & $63(15.75)$ & $18(11.84)$ & $45(18.15)$ & 18 & 21 & 2 \\
\hline $40-49$ & $39(9.75)$ & $15(9.87)$ & $24(9.68)$ & 5 & 12 & 0 \\
\hline$\geq 50$ & $56(14.00)$ & $13(8.55)$ & $43(17.34)$ & 8 & 11 & 0 \\
\hline
\end{tabular}


Table 2: Clinical and demographic characteristics of febrile patients stratified by chikungunya and malaria infections

\begin{tabular}{|c|c|c|c|c|}
\hline Characteristics & No. of subjects $(n=400)$ & $\begin{array}{l}\text { CHIKV } \\
(n=9)\end{array}$ & $\begin{array}{l}\text { CHIKV } \\
(n=88)\end{array}$ & Malaria $(n=112)$ \\
\hline \multicolumn{5}{|l|}{ Sex } \\
\hline Male & $152(38.00 \%)$ & $1(11.11 \%)$ & $25(28.41 \%)$ & $35(31.25 \%)$ \\
\hline Female & $248(62.00 \%)$ & $8(88.89 \%)$ & $63(71.59 \%)$ & $77(68.75 \%)$ \\
\hline \multicolumn{5}{|c|}{ Clinical characteristics } \\
\hline Headache & $276(69.00 \%)$ & $5(55.56 \%)$ & $55(62.50 \%)$ & $82(73.21 \%)$ \\
\hline Nausea & $221(55.25 \%)$ & $2(22.22 \%)$ & $14(15.91 \%)$ & $56(50.00 \%)$ \\
\hline Vomiting & $246(61.50 \%)$ & $1(11.11 \%)$ & $4(4.55 \%)$ & $63(56.25 \%)$ \\
\hline Joint ache & $273(68.25 \%)$ & $8(88.89 \%)$ & $74(84.10 \%)$ & $44(39.29 \%)$ \\
\hline Stiff neck & $135(33.75 \%)$ & $0(0.00 \%)$ & $5(5.68 \%)$ & $3(2.68 \%)$ \\
\hline Seizures & $141(35.25 \%)$ & $0(0.00 \%)$ & $0(0.00 \%)$ & $8(7.14 \%)$ \\
\hline Leg weaknesses & $238(59.50 \%)$ & $4(44.44 \%)$ & $49(55.68 \%)$ & $66(58.93 \%)$ \\
\hline Skin rash & $132(33.00 \%)$ & $6(66.67 \%)$ & $68(77.27 \%)$ & $2(1.79 \%)$ \\
\hline Abdominal pains & $167(41.75 \%)$ & $0(0.00 \%)$ & $0(0.00 \%)$ & $33(29.46 \%)$ \\
\hline Pallor & $217(54.25 \%)$ & $0(0.00 \%)$ & $1(1.14 \%)$ & $26(23.21 \%)$ \\
\hline Jaundice & $226(56.50 \%)$ & $0(0.00 \%)$ & $0(0.00 \%)$ & $2(1.79 \%)$ \\
\hline Laboured breathing & $232(58.00 \%)$ & $0(0.00 \%)$ & $1(1.14 \%)$ & $14(12.50 \%)$ \\
\hline
\end{tabular}

\section{Malaria and Chikungunya infections}

Out of 400 clinical specimens analysed, 116 tested positive for malaria by mRDT, 96.55\% (112) were confirmed by microscopy. Chikungunya specific antibodies were detected in 97 patients, out of which $83(20.75 \%)$ tested IgG positive whilst 14 (3.50\%) tested IgM positive only (Table 2). Out of 97 CHIKV seropositive subjects, 89 (91.75\%) subjects had no malaria. Chikungunya and malaria coinfection was found to be 7.14\% (8/112). The highest seropositivity was in the 20 to 29 years old group accounting for $53.61 \%(n=52)$ seropositive CHIKV febrile patients (Table 1$)$. The proportion of participants who were IgG seropositive was higher than the proportion of participants who were IgM seropositive for all age groups (Table 2). High frequency of malaria and chikungunya infections was recorded among females. Within the group infected with chikungunya alone, females had infection rate of $73.20 \%$ whilst males had $26.80 \%$ (Figure 1 ).

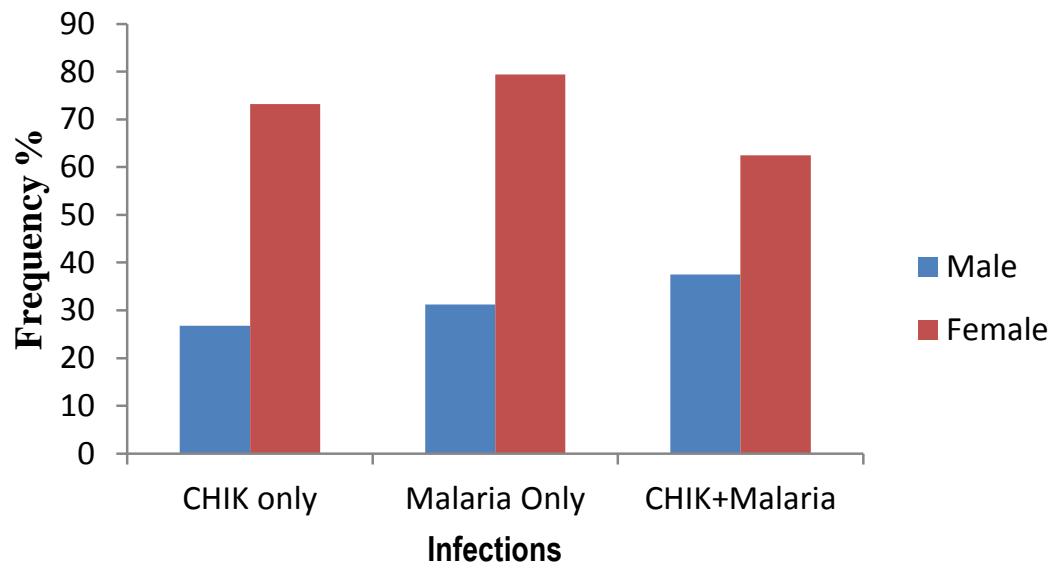

Figure 1: Frequency of chikungunya and malaria infections by sex

\section{Discussion}

Chikungunya is among the febrile illnesses in patients seeking health care in Karagwe district. Chikungunya-malaria co-infection was prevalent in the area. Patients with malaria and CHIKV infections were clinically manifested by related clinical characteristics. In malaria endemic areas 
most febrile illnesses have been and are still treated as malaria cases due to lack of diagnostic capacity to properly rule-out malaria as a cause of fever and as well, identify alternative fever causative agents (Chipwaza et al., 2014; Sow et al.,2016; Kajeguka et al., 2017).

The proportion of chikungunya seropositivity was not comparable between febrile patients, indicating variations in the exposure among age groups. The IgG positivity was significantly higher in adults than in children. The increase of IgG seropositivity indicates previous exposure to CHIKV among adults. The seroprevalence of $24.25 \%$ observed in this study is four times as higher than that (4.7\%) previously reported in central Tanzania (Chipwaza et al., 2014). The observed variations in the prevalence of chikungunya between different studies may be attributed to geographical zones where the studies were done, targeted age groups, seasonal variations, agricultural activities and time factor (Hertz et al., 2012; Crump et al., 2013; Weller et al., 2014; Kajeguka et al 2017). Previous studies have also indicated the occurrence of presumptive and prior exposure to CHIKV infections in the country (Chipwaza et al., 2014; Kajeguka et al., 2016). Our results showed that anti-CHIKV IgM antibodies were found among a very few patients within 7 days after the onset of fever hence suggesting possibility of acute infection (presumptive acute CHIKV infections). Most often, in acute CHIKV infections IgM antibodies appear first and can be detected during the first week of the disease particularly from day 5 while IgG antibodies appear shortly afterwards (Chipwaza et al., 2014). The detection of IgM antibodies in patients who had a history of fever for less than 5 days, possibly indicate a recent previous CHIKV exposure rather than acute infection.

Age and sex are proxy-factors for specific behaviour that cause higher exposure to mosquito bites. Outdoor stays during the daytime and dusk favours bite by Aedes mosquitoes. These practices and lifestyle of adults whose ages range 20-39 years may make them be vulnerable to these mosquito-borne arbovirus diseases (Sow et al.,2016; Furuya-Kanamori et al.,2016), unlike in malaria transmission (Mboera et al., 2007; Moshi et al., 2017). Females indicated the highest infection rate of the two infections than their counterpart males. Owing to low immunity in lactation and pregnancy, these physiological differences may contribute to different susceptibility levels (Sow et al.,2016).

The study has drawn a significant association between clinical characteristics and the probability of a positive diagnosis of chikungunya and malaria. These findings are in line with other studies that reported joint pain, headache, nausea, vomiting, myalgia, and skin rash were associated with chikungunya and malaria infections (Mtove et al., 2011; Chipwaza et al., 2014; Furuya-Kanamori et al.,2016). However, these symptoms are common and non-specific, and thus could be mistakenly identified as caused by a variety of other febrile illnesses such as malaria, typhoid, dysentery, and bacterial meningitis and pose a challenge to differential diagnosis. Therefore, it is recommended to establish a better means of diagnosis of fever to circumvent mistreatment of medical important diseases.

The study has some limitations. Since this study was cross-sectional, a cautious interpretation of this finding is necessary, particularly in the absence of comparison between acute and convalescent serum from the same patient. Another limitation was inability to perform molecular technique such as reverse transcriptase-Polymerase chain reaction (PCR) analysis for CHIKV due to lack of positive controls for the test.

In conclusion, chikungunya and malaria infections exist in all age groups of febrile patients seeking health care in Karagwe district. Since there is no routine diagnosis of mosquito-borne viral diseases in Tanzanian health facilities, it is likely that such infections go unnoticed or when causing fever end up being treated as other common infections such as malaria, bacterial or fungal infections. 


\section{Acknowledgments}

This work was supported by Wellcome Trust through Southern African Centre for Infectious Disease Surveillance (SACIDS) coordination Unit of the Sokoine University of Agriculture, Project Number: Grant WTo87546MA.

\section{Competing interests}

Authors have declared that no competing interest.

\section{References}

Bisimwa, N.P., Kinimi, E., Shayo, J.M., Angwenyi, O.S., Weyer, J., Jansen van Vuren, P., Paweska, T.J., Mboera, L.E.G., Rweyemamu, M.M., Misinzo, G. \& Kasanga, J. C.(2018) Distribution and diversity of mosquitoes and the role of Aedes in transmission of arboviruses in selected districts of Tanzania. International journal of mosquito Research 5:53-60.

Carlstedt, A. (1997) Malaria catastrophe in East Africa. Lancet 350(9085):1180.

CDC (2016) Centers for Disease Control and Prevention: Chikungunya geographic distribution. Available from: https://www.cdc.gov/chik/geo

Chipwaza, B., Mugasa, J. P., Selemani, M., Amuri, M., Mosha, F., Ngatunga, S. D. \& Gwakisa, P. S. (2014) Dengue and Chikungunya Fever among Viral Diseases in Outpatient Febrile Children in Kilosa District Hospital, Tanzania. PLoS Neglected Tropical Diseases 8 (11): e3335.

Crump, J.A., Morrissey, A.B., Nicholson, W.L., Massung, R.F., Stoddard, R.A., Galloway, R.L., Ooi, E.E., Maro, V.P., Saganda, W., Kinabo, G.D., Muiruri, C. \& Bartlett, J.A. (2013) Etiology of Severe Non-malaria Febrile Illness in Northern Tanzania: A Prospective Cohort Study. PLoS Neglected Tropical Diseases 18:7(7):e2324.

D'Acremont, V., Kilowoko, M., Kyungu, E., Philipina, S., Sangu, W., Kahama-Maro, J., Alamo, L., Cherpillod, P., Lengeler, C., Kaiser, L. \& Genton, B. (2014) Beyond malaria, causes of fever in outpatient Tanzanian children. New England Journal of Medicine 370: 809-817.

Deller, J.J \& Russell, P.K. (1968) Chikungunya disease: American Journal of Tropical Medicine and Hygiene 17: 107-111.

Faustine, N.L., Sabuni, J.E., Ndaro, J.A., Paul, E. \& Chilongola, O.J. (2017) Chikungunya, Dengue and West Nile virus Infections in Northern Tanzania. Advances in Medicine and Medical Research 24 (4).

Furuya-Kanamori, L., Liang, S., Milinovich, G., Soares Magalhaes, R.J., Clements, A.C.A., Hu W., Brasil, P., Frentiu, D.F., Dunning, R. \& Yakob, L. (2016) Co-distribution and coinfection of chikungunya and dengue viruses. BMC Infectious Diseases 16: 1.

Hercik, C., Cosmas, L., Mogeni, O.D., Wamola, N., Kohi, W., Omballa, V., Ochieng, M., Lidechi, S., Bonventure, J., Ochieng, C., Onyango, C., Fields, B.S., Mfinanga, S. \& Montgomery, J.M. (2017) A diagnostic and epidemiologic investigation of acute febrile illness (AFI) in Kilombero, Tanzania. PLoS ONE 12(12):e0189712.

Hertz, J.T., Munishi, O.M., Ooi, E.E., Howe, S., Lim, W.Y., Chow, A., Morrissey, A.B., Bartlett, J.A., Onyango, J.J., Maro, V.P., Kinabo, G.D., Saganda, W., Gubler, D.J. \& Crump, J.A.. (2012) Chikungunya and dengue fever among hospitalized febrile patients in northern Tanzania. American Journal of Tropical Medicine and Hygiene 86: 171-177.

Kajeguka, C. D., Kaaya, D.R., Mwakalinga, S., Ndossi, R., Ndaro, A., Chilongola, O.J., Mosha, W.F., Schiøler, L.K., Kavishe, A.R. \& Alifrangis, M (2016) Prevalence of dengue and chikungunya virus infections in north-eastern Tanzania: a cross sectional study among participants presenting with malaria-like symptoms. BMC Infectious Diseases 16:183. 
Kajeguka, D.C., Descrochers, R.E., Mwangi, R., Mgabo, M.R., Alifrangis, M., Kavishe, R.A., Mosha, F.W. \& Kulkarni, M.A. (2017) Knowledge and practice regarding dengue and chikungunya, a cross-sectional study among Healthcare workers and community in Northern Tanzania. Tropical Medicine \& International Health 22: 583-593.

Ligon, B.L.(2006) Reemergence of an Unusual Disease: The Chikungunya Epidemic. Seminars in Pediatric Infectious Diseases 17:99-104.

Lindsay, S.W., Bodka, R., Malima, R., Msangeni, H.A. \& Kissinza, W. (2000) The effect of the 199798 El Nino on highland malaria in Tanzania. Lancet 355:989-990.

Lumsden, W.H. (1955) An epidemic of virus disease in southern province, Tanganyika territory, in 1952-53. II. General description and epidemiology. Transactions of the Royal Society of Tropical Medicine and Hygiene 49:33-57.

Mashauri, F.M., Kinung'hi, S.M., Kaatano, G.M., Magesa, S.M., Kishamawe, C., Mwanga, J.R., Nnko, S.E., Malima, R.C., Mero, C.N. \& Mboera L.E. (2013) Impact of indoor residual spraying of lambda-cyhalothrin on malaria prevalence and anemia in an epidemic-prone district of Muleba, north-western Tanzania. American Journal of Tropical Medicine and Hygiene 88:841-849

Mboera, L.E.G., Mazigo, H.D., Rumisha, S.F. \& Kramer, R.A. (2013) Towards malaria elimination and its implication for vector control, disease management and livelihoods in Tanzania. MalariaWorld Journal 4(19):18-20.

Mboera, L.E.G. \& Kitua, A.Y. (2001) Malaria epidemics in Tanzania: African Journal of Health Sciences 8:14-18.

Mboera, L.E.G., Makundi, E.A. \& Kitua, A.Y.(2007) Uncertainty in malaria control in Tanzania: Crossroads and challenges for future interventions. American Journal of Tropical Medicine and Hygiene 77(Suppl. 6):112-118.

Mohan, A., Kiran, D.H., Manohar, I.C. \& Kumar, D.P. (2010) Epidemiology, clinical manifestations, and diagnosis of chikungunya fever: lessons learned from the re-emerging epidemic. Indian Journal of Dermatology 55:54-63.

Moshi, I.R., Ngowo, H., Dillip, A., Msellemu, D., Madumla, E.P., Okumu, F.O., Coetzee, M., Mnyone, L.L. \& Manderson, L. (2017) Community perceptions on outdoor malaria transmission in Kilombero Valley, Southern Tanzania. Malaria Journal 16:274.

Mtove, G., Amos, B., Nadjm, B., Hendriksen, I.C.E., Dondorp, A.M., Mwambuli, A., Kim, D.R., Ochiai, R.L., Clemens, J.D., von Seidlein, L., Reyburn, H. \& Deen J. (2011) Decreasing incidence of severe malaria and community-acquired bacteraemia among hospitalized children in Muheza, north-eastern Tanzania, 2006-2010. Malaria Journal 10:320.

Ndosi, R., Kwigizile, E., Ibrahim, U., Dossajee, U., Rwiza, J., Kabanyana, C., Ndaro, A. \& Chilongola, J. (2016) Risk Factors for Concurrent Malaria and Arbovirus Infections in Handeni, Northeastern Tanzania. International Journal of Tropical Disease \& Health 20: 1-7.

O'Meara, W.P., Bejon, P., Mwangi, T.W., Okiro, E.A., Peshu, N., Snow, R.W., Newton, C.R. \& Marsh, K. (2008) Effect of a fall in malaria transmission on morbidity and mortality in Kilifi, Kenya. Lancet 372:1555-1562.

Robinson, M.C. (1955) An epidemic of virus disease in Southern province, Tanganyika Territory, in 1952-53. Clinical features. Transactions of the Royal Society of Tropical Medicine and Hygiene 49: 28-32.

Sow, A., Loucoubar, C., Diallo, D., Faye, O., Ndiaye, Y., Senghor, C.S., Dia, A.T., Ousmane, F., Weaver, S.C., Diallo, M., Malvy, D. \& Sall, A. A. (2016) Concurrent malaria and arbovirus infections in Kedougou, southeastern Senegal. Malaria Journal 15:47(1).

Thiberville, S.D., Moyen, N., Dupuis-Maguiraga, L., Nougairede, A., Gould, E.A., Roques, P., de Lamballerie, X. (2013) Chikungunya fever: epidemiology, clinical syndrome, pathogenesis and therapy. Antiviral Research Journal 99:345-370.

Weller, N., Clowes, P., Dobler, G., Saathoff, E., Kroidl, I., Ntinginya, N. E., Maboko, L., Löscher, T., Hoelscher, M. \& Heinrich, N. (2014) Seroprevalence of Alphavirus Antibodies in a Cross- 
Sectional Study in Southwestern Tanzania Suggests Endemic Circulation of Chikungunya. PLoS Neglected Tropical Diseases 8(7): e2979. 